\title{
Computational Screening of Cationic Collectors Adsorption on the Mica (001) Surface
}

\author{
Dan Su, ${ }^{1, a}$, Yuelong $\mathrm{Liu}^{1, \mathrm{~b},{ }^{*}}$ and Gousheng $\mathrm{Liu}^{2, \mathrm{c}}$ \\ ${ }^{1}$ School of Chemistry and Chemical Engineering, Jiangxi Science \& Technology Normal University, \\ Nanchang 330013, Jiangxi Province, China. \\ ${ }^{2}$ State Key Laboratory of Chemical Engineering, East China University of Science and Technology, \\ Shanghai 200237, China \\ asuzihan1234@126.com, *,blylgyx@163.com, 'gsliu@ecust.edu.cn \\ * corresponding author: Yuelong Liu
}

Keywords: Molecular dynamics simulation; Collector cations; Mica; Interaction energy; S order parameter.

Abstract. Molecular dynamics simulations have been performed for the study of different cationic collectors adsorption on the mica (001) surface in the vacuum. The relationship between structure of ammonium ions and the flotation performance of mica has been discussed. The $\mathrm{S}$ order parameter is further calculated to investigate the discrepancy of adsorption orientation in different systems.

\section{Introduction}

Collectors play an extremely important role in the separation of finely ground valuable minerals from their associated gangue via flotation. As typical cationic collectors, amines have been widely applied in the flotation. With the rapid development of computer technology, computer simulation has become a bridge between theory and experiments. Du et al.[1] studied the adsorption of dextrin and the surfactant dodecyl trimethyl ammonium bromide at the talc mineral surfaces to reveal the configuration of adsorbed molecules at the edge surface and the basal surface of talc by molecular dynamics simulation. It was found that the hydrophobic chains of DTAB prederentially adsorbed at the basal surface through hydrophobic interactions and electrostatic interactions played a significant role in DTAB adsorption at the talc edge surface. Wang et al.[2] used MD simulations to investigate the adsorption of sodium oleate (NaOL), dodecylamine hydrochloride (DDAH)and the mixture of them on the muscovite surface in the aqueous solution. The results suggested that sodium oleate molecules could not independently adsorb on the surface and dodecylamine hydrochloride molecules played an important role in the adsorption of the mixture of the two ions on the muscovite surface.

In this work, in order to explain interaction discrepancy of different cationic collectors on the mica surface, the adsorption of alkyl propyl ether ammonium, N-alkyl-1,3-diaminopropane, $\mathrm{N}, \mathrm{N}$-dimethyl-alkyl ammonium, alkyl dimethyl benzyl ammonium with different alkyl chain lengths on the mica (001) surface are investigated employing molecular dynamics simulations with PCFF_phyllosilicates force field [3].

\section{Simulation details}

Models. The supercell model of Mica is prepared according to ${ }^{29} \mathrm{Si}$ NMR data.The double layer mica (001) surface is built by cleaving the (001) surface at the middle of the interlayer. The models of the ammonium ions $\mathrm{C}_{\mathrm{n}} \mathrm{H}_{2 \mathrm{n}+1}-\mathrm{O}\left(\mathrm{CH}_{2}\right)_{3} \mathrm{NH}_{3}{ }^{+}, \quad \mathrm{C}_{\mathrm{n}} \mathrm{H}_{2 \mathrm{n}+1}-\mathrm{N}\left(\mathrm{CH}_{2}\right)_{3} \mathrm{NH}_{3}{ }^{+}, \quad \mathrm{C}_{\mathrm{n}} \mathrm{H}_{2 \mathrm{n}+1}-\mathrm{N}\left(\mathrm{CH}_{3}\right)_{2} \mathrm{H}^{+}$, $\mathrm{C}_{\mathrm{n}} \mathrm{H}_{2 \mathrm{n}+1}-\mathrm{N}\left(\mathrm{CH}_{3}\right)_{2}\left(\mathrm{C}_{7} \mathrm{H}_{7}\right)^{+}$with alkyl chain lengths $\mathrm{n}=6,8,10, \ldots, 22$ are constructed using the graphical interface of Materials Studio 6.1. Dmol3 module is employed for optimizing the geometries of ammonium ions and calculating the ESP charges. A total of 36 different models of the ammonium ions are placed over the mica (001) surface employing Amorphous Cell module and the build Layer tool. For the ammonium ion, the positions in the $\left[\mathrm{Si}_{4} \mathrm{Al}_{2}\right]$ di-trigonal cavity is preferential adsorption site rather than the $\left[\mathrm{Si}_{5} \mathrm{Al}_{1}\right]$ cavity, which is shown in the earlier simulation work[4]. Therefore, for all initial configurations, the $\mathrm{N}$-containing polar head groups of all collector cations are positioned above 
the location of $\left[\mathrm{Si}_{4} \mathrm{Al}_{2}\right]$ hexagonal cavities on the mica (001) surface and the hydrophobic chains face the vacuum in our simulations.

Simulations. After a 100-step energy minimization with smart minimizer method proceeded to eliminate the unreasonable contacts, the MD simulations are carried out in the NVT ensemble. The temperature is fixed at $298 \mathrm{~K}$ controlled by the Nose-Hoover-langevin (NHL) thermostat. The long-range electrostatic interactions are treated using the Ewald summation method with an accuracy of $10^{-3}$, and the non-bond cutoff distance for van der Waals interactions is set to $12.5 \AA$. Total simulation time for all systems is $3.0 \mathrm{~ns}$. After $2.5 \mathrm{~ns}$ of equilibration, the equilibrium dynamic trajectories of the last $500 \mathrm{ps}$ are used for data analysis at $20 \mathrm{fs}$ intervals.

\section{Results and Discussion}

ESP charges of head groups. The calculated ESP charges of N-containing polar head groups of ammonium ions with alkyl chain lengths $n=6,8,10, \ldots, 22$ are shown in Fig. 1.

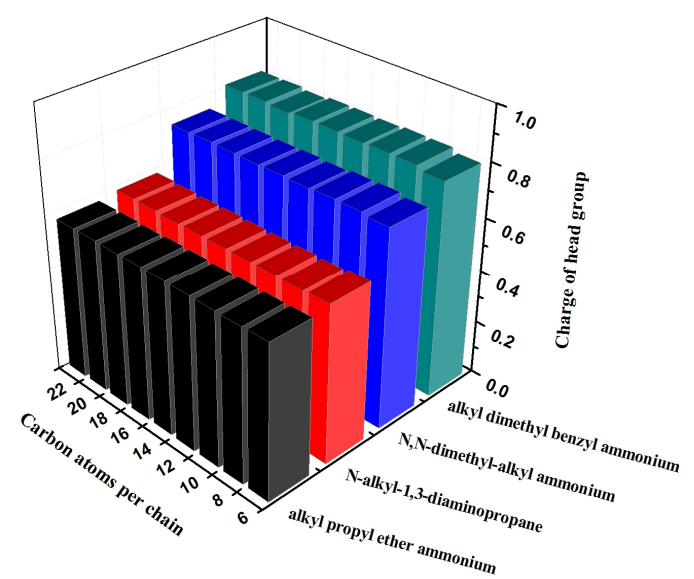

Fig. 1. the ESP charges of head groups of different ammonium ions

As illustrated in Fig. 1, for a homologous series, the ESP charges of head groups have almost no differences when the number of carbon atoms in alkyl chain increases from 6 to 22, which indicates that the effect of alkyl chain length on the atomic charge distribution of the collector cations is limited. However, for different types of alkylammonium, ESP charges of polar head groups follow the order of alkyl propyl ether ammonium, N-alkyl-1,3-diaminopropane < N,N-dimethyl-alkyl ammonium < alkyl dimethyl benzyl ammonium, which suggests that both substitution of $\mathrm{N}$-containing polar group and heteroatom in the alkyl chain have a major influence on the atomic charge distribution. The results show that the charge distribution between the atoms is affected by the structure of the ammonium ions rather than chain length. In Fig. 1, the values of ESP charges are all positive, indicating that the $\mathrm{N}$-terminal part of ammonium ions can adsorb on negatively charged mica surface.

Interaction energy. The interaction energy is used to quantify the relative affinity of regent - mineral surface interactions[5], and can be calculated using the following formula:

$$
E_{\text {interaction }}=E_{\text {total }}-\left(E_{\text {surface }}+E_{\text {regent }}\right)
$$

Where $E_{\text {total }}$ is the energy of the surface and the regent, $E_{\text {surface }}$ is the energy of the surface without the regent and $E_{\text {regent }}$ is the energy of the regent without the surface. As an excellent measure of the strength of interactions between the regents and the mineral surface, it can be considered as the judgment of the collecting ability of regents in mineral flotation. According to the method, the interaction energies between different collector cations and the mica (001) surface are calculated, and the obtained values are presented in Fig. 2. 


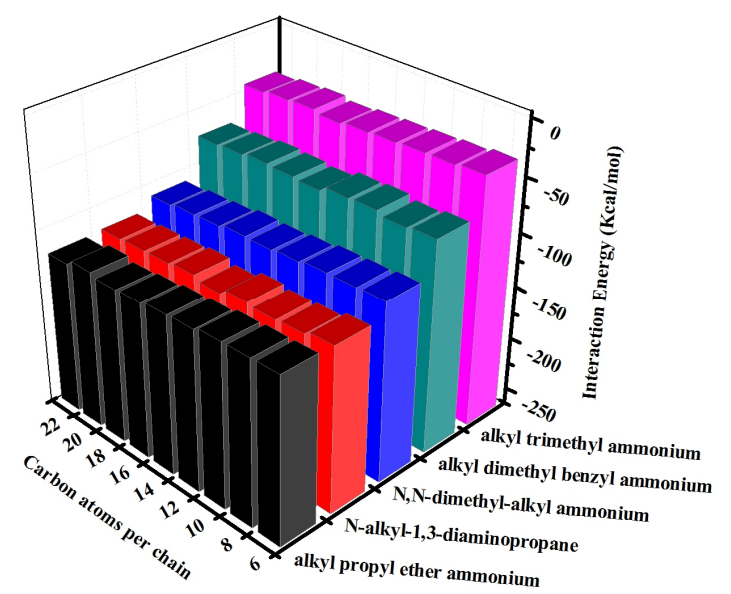

Fig. 2.Computed interaction energies between different ammonium ions and the mica (001) surface

As depicted in Fig.2, the interaction energies of these cationic collectors with chain lengths ranging from $\mathrm{C}_{6}$ to $\mathrm{C}_{22}$ have small deviations, indicating that hydrocarbon chain length have a limited influence on the interaction energy. Meanwhile, the calculated interaction energies of alkyl propyl ether ammoniums on the mica (001) surface are lower than that of alkyl trimethyl ammoniums with the mica surface[6], indicating that the strength of alkyl propyl ether ammonium-mica surface interactions excels the strength of alkyl trimethyl ammonium - mica surface interactions. Therefore, compared with alkyl trimethyl ammonium, alkyl propyl ether ammonium is much more effective collector for mica flotation, which is consistent with the results drawn from the flotation experiments of these cationic collectors onto mica in the earlier work [7]. In our MD simulation, quantitative result is obtained by calculating the interaction energy of one collector cation with the mica surface in the vacuum enviroment and the water molecules are not considered. However, it is to be noted that in spite of this apparent lacuna in the process of simulations, the results of the MD simulations which model collector-mineral surface interactions are in good agreement with the experimental results with respect to collecting abilities of these cationic collectors on mica surfaces. Such a reasonable match between computational and experimental data demonstrate the power of our computational data in being able to estimate the interaction strength between the collectors and mica correctly, which can explain the discrepancies including the collecting abilities and adsorbing power of these cationic collectors. Moreover, designing or screening out the highly selective and appropriate collectors for the minerals flotation can be achieved by quantification of the interactions between collectors and mineral surface.

We can find that the values of interaction energies follow the order of alkyl propyl ether ammonium, $\mathrm{N}$-alkyl-1,3-diaminopropane < N,N-dimethyl-alkylammonium < alkyl dimethyl benzyl ammonium in Fig.2. That is to Say, both $\mathrm{N}$-alkyl-1,3-diaminopropane and alkyl propyl ether ammonium interact more strongly with the mica (001) surface as compared to N,N-dimethyl-alkyl ammonium or alkyl dimethyl benzyl ammonium, implying that collecting power of the alkyl propyl ether ammonium and $\mathrm{N}$-alkyl-1,3-diaminopropane is better than that of $\mathrm{N}, \mathrm{N}$-dimethyl-alkyl ammonium and alkyl dimethyl benzyl ammonium. By comparing the interaction energy, it is found that both alkyl propyl ether ammonium and $\mathrm{N}$-alkyl-1,3-diaminopropane should be the more appropriate collectors for mica flotation.

$\mathbf{S}$ order parameter. To describe the orientation of collector cations with respect to the mica basal surface, the $S$ order parameter [8] is further calculated.

As depicted in Fig. 3, the value of local $S_{1}$ order parameter decrease when the number of substitutions in $\mathrm{N}$-containing polar group increases from 0 to 3 . For alkyl propyl ether ammonium and $\mathrm{N}$-alkyl-1,3-diaminopropane cations, the $\mathrm{CH}_{2} \mathrm{NH}_{3}{ }^{+}$part can be closely attached to the mica surface and is almost perpendicular to the mica (001) surface, due to the formation of hydrogen bonds between head group $\mathrm{H}$ atoms and surface bridging oxygen atoms. By contrast, average order 
parameters $\mathrm{S}_{1}$ of $\mathrm{N}, \mathrm{N}$-dimethyl-alkyl ammonium and alkyl dimethyl benzyl ammonium are negative. As a spacer, the methyl and bulky benzyl substituents bonding to $\mathrm{N}$ atom in the polar head group result in the parallel orientation of the head group of alkyl dimethyl benzyl ammonium relative to the mica (001) surface.

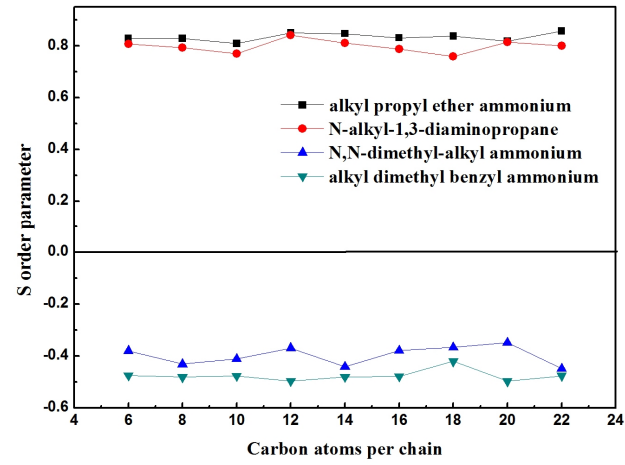

Fig. 3.The local $\mathrm{S}_{1}$ order parameter

\section{Conclusions}

In this paper,the calculated interaction energies reveal that collecting power of alkyl propyl ether ammonium for mica is better than that of alkyl trimethyl ammonium, which match well with those experiments in the earlier work. Calculated results also show that the strength of collector-mineral surface interactions follows the order of alkyl propyl ether ammonium, $\mathrm{N}$-alkyl-1,3-diaminopropane $<\mathrm{N}, \mathrm{N}$-dimethyl-alkyl ammonium < alkyl dimethyl benzyl ammonium, indicating that the alkyl propyl ether ammonium and $\mathrm{N}$-alkyl-1,3-diaminopropane are much more effective collectors than $\mathrm{N}, \mathrm{N}$-dimethyl-alkyl ammonium and alkyl dimethyl benzyl ammonium for mica flotation.

\section{Acknowledgements}

This work was sponsored by the National Science Foundation of China (No. 51164009).

\section{References}

[1] H. Du and J. D. Miller: International Journal of Mineral Processing. Vol. 84 (2007), p. 172

[2] L.Wang,Y.Hu, W.Sun and Y.Sun: Applied Surface Science. Vol. 327 (2015), p. 364

[3] H. Heinz, H. Koerner, K.L. Anderson, R.A. Vaia and B. Farmer: Chemistry of materials. Vol. 17 (2005), p. 5658

[4]M.Odelius, M.Bernasconi and M.Parrinello: Physical Review Letters. Vol. 78 (1997), p.2855

[5] B.Rai, P.Sathish, J.Tanwar, K.Moon and D. Fuerstenau: Journal of Colloid and Interface Science. Vol. 362 (2011), p. 510

[6]Y. Xu, Y.L. Liu, D.D. He and G.S. Liu: Minerals Engineering. Vol. 53 (2013), p. 101

[7] Z.Y Gao: Journal of Bgrimm. Vol. 1(1992), p. 60 (In Chinese)

[8] E. Egberts and H. J. C. Berendsen: Journal of Chemical Physics. Vol. 89 (1988), p. 3718 